\title{
La conectividad funcional entre el estriado derecho e izquierdo en estado de reposo se asocia a sensibilidad a la recompensa
}

\author{
FCO. JAVIER LÓPEZ FERNÁNDEZ \\ al225790@uji.es \\ CÉSAR ÁVILA RIVERA \\ avila@uji.es
}

\section{Resumen}

Introducción: La Teoría de la Sensibilidad al Refuerzo sostiene que las diferencias en la reactividad de determinados sistemas cerebrales que controlan los procesos motivacionales, emocionales y de aprendizaje subyacen a las diferencias individuales de los rasgos de personalidad básicos. Uno de estos sistemas es el sistema de activación conductual que se encarga de responder ante estímulos apetitivos y de omisión de castigos, relacionados con la conducta de aproximación. Estudios recientes han demostrado que los individuos con una mayor actividad crónica del sistema de activación conductual muestran un menor volumen de sustancia gris en los ganglios basales y en el córtex prefrontal medial (Barrós-Loscertales y cols., 2006). El objetivo del presente trabajo es comprobar si estas diferencias anatómicas llevan asociadas un distinto patrón de conectividad funcional. Metodología: Mediante la técnica de resonancia magnética funcional en estado de reposo (RMF-ER), hemos investigado en estudiantes varones si existen diferencias en conectividad funcional entre esas áreas cerebrales relacionadas con la sensibilidad a la recompensa. Resultados: Los resultados mostraron que las puntuaciones en sensibilidad a la recompensa se asocian a una mayor conectividad funcional entre el estriado izquierdo y derecho. Discusión: Estos datos reflejan una diferencia funcional basada en la personalidad en zonas cerebrales directamente asociadas al procesamiento de la recompensa.

Palabras clave: sistema de activación conductual (SAC), estriado, ganglios basales, estado de reposo y sensibilidad a la recompensa.

\footnotetext{
Abstract

Introduction: The Reinforcement Sensitivity Theory sustains that differences in the reactivity of certain brain systems which control motivational, emotional and learning processes underlie individual differences in basic personality traits. These systems are responsible for controlling behaviours and specific emotions that might be related to certain cognitions. One of these systems is the behavioural activation system, which is
} 
responsible for responding to appetitive stimulus and omission of punishment. This system is linked to approach behaviours. Recent studies have proved that individuals with greater chronicle activity in the behavioural activation system present a smaller volume of grey matter in bilateral basal ganglia and the medial prefrontal cortex (Barrós-Loscertales et al., 2006). The aim of this study is to check whether these anatomical differences are related to a different pattern of functional connectivity. Method: By technique functional magnetic resonance imaging in the resting state (fMRI-ER), we studied if functional connectivity differences exist in male students between these brain areas associated with sensitivity to reward. Results: The results showed sensitivity to reward marks are linked to increased functional connectivity between the left and the right striatum. Discussion: Our data show a functional difference in personality terms in areas directly related to reward processing.

Keywords: behavioural activation system (BAS), striatum, basal ganglia, resting state, sensitivity to reward.

\section{Introducción}

Al comienzo de los años 70 Gray (1970) formuló la Teoría de la Sensibilidad al Refuerzo (RST, «Reinforcement Sensitivity Theory»). Este hecho supuso una revolución dentro del estudio biológico de la personalidad, ya que propuso que las diferencias individuales en los distintos rasgos de personalidad provenían de variaciones en la reactividad de sistemas neuroconductuales subyacentes a procesos motivacionales, emocionales y de aprendizaje (Depue y Collins, 1999). EI modelo RST postula la existencia de diversos sistemas neurales especializados en detectar, procesar y responder ante determinados estímulos. Así, el tipo de estímulo pondrá en marcha un determinado sistema neural, generando estados motivacionales, emocionales, respuestas conductuales y aprendizajes diferentes en función de su actividad (Corr, 2008a). Según el modelo, diversos sistemas cerebrales específicos se encargarían de controlar conductas y emociones concretas que se podrían asociar a determinadas percepciones o cogniciones (Corr, 2008b). Estos sistemas locales estarían asociados entre sí para funciones más generales en contextos más amplios. Al mismo tiempo, estos sistemas interaccionarían entre ellos y estarían modulados por sistemas generales (como los de arousal y atención). De este modo, la personalidad podría entenderse como el resultado de las diferencias individuales en la reactividad de diferentes sistemas neurales.

El sistema de activación conductual (BAS, según sus siglas en inglés), surgido de la RST, es un sistema cerebral encargado de responder ante estímulos condicionados e incondicionados apetitivos y de omisión de castigos. Cuando uno de estos estímulos está presente se dan dos efectos en la conducta que están mediados por el BAS, un efecto motivacional, debido a un incremento en el arousal que estimula y redirige la conducta hacia la fuente de reforzamiento; y un efecto en el aprendizaje, debido a que se redirige la atención sobre el estímulo de recompensa, facilitando el procesamiento de la información y el aprendizaje de relaciones estímulo-estímulo y estímulo-respuesta (Pickering y Gray, 2001; Pickering y Smillie, 2008). Para evaluar el BAS empleamos la escala de sensibilidad a la recompensa (SR), basada en la medición de conductas dirigidas a la obtención de refuerzos como dinero, sexo, búsqueda de sensaciones, etc. (Torrubia, Ávila, Moltó y Caseras; 2001). Esta escala mide tanto diferencias individuales en sensibilidad a la recompensa (frecuencia con la que se detectan estímulos condicionados apetitivos) como 
reactividad a esos estímulos, es decir, intensidad de las conductas de aproximación y búsqueda de recompensa.

Generalmente se propone al circuito dopaminérgico y a las áreas asociadas como parte principal del sistema de activación conductual (Haber y Knutson, 2010; Berridge y Kringelbach, 2008). Estas áreas forman una red que incluye el sistema orbitofrontal, el cortex prefrontal medial, el cuerpo estriado y la amígdala (McClure, York y Montague, 2004). Diversos estudios han puesto de manifiesto la importancia del estriado en el BAS y, por lo tanto, en la sensibilidad a la recompensa (Costumero, 2013). Mediante estudios de morfología usando resonancia magnética se ha observado que el volumen del estriado ventral correlaciona negativamente con las puntuaciones en SR (Barrós-Loscertales y cols., 2006). También en estado de reposo se encontró una mayor aleatoriedad de las series temporales del estriado ventral en sujetos con alta SR (Hahn y cols., 2012).

La conectividad funcional se define como la dependencia temporal de los patrones de actividad neuronal de regiones anatómicamente separadas del cerebro (Aertsen, Gerstein, Habib y Palm, 1989; Friston, Frith, Liddle y Frackowiak, 1993). En los últimos años, un creciente número de estudios de neuroimagen han comenzado a explorar la conectividad funcional mediante la medición de niveles de co-activación en imágenes de resonancia magnética funcional en estado de reposo entre regiones cerebrales anatómicamente separadas (Biswal, Yetkin, Haughton y Hyde, 1995; Salvador y cols., 2005). Estos estudios han aportado importantes conocimientos en la organización de la comunicación funcional en las redes cerebrales. Harmelech y Malach (2013) propusieron que los patrones de correlación entre las fluctuaciones de actividad espontánea (FES) durante el estado de reposo ( «resting state») reflejarían el perfil, a priori, de los sesgos cognitivos, o tendencias individuales, codificados como eficacia sináptica en redes corticales. Por lo tanto, las FEs ofrecen un nuevo medio para el mapeo de las diferencias individuales de rasgos como los de personalidad, tanto en sujetos normales como en muestras clínicas. Se presentaron tres hipótesis para avalar esta idea: 1) las FEs corresponde a patrones de activación que se producen durante la ejecución de tareas típicas en el día a día; 2) las diferencias individuales y grupales en sesgos deberían reflejarse en distintos patrones espontáneos de activación; y finalmente, 3) las FEs pueden ser activamente remodeladas a largo plazo mediante un intenso y localizado entrenamiento cortical.

El objetivo de nuestra investigación es analizar la relación entre las diferencias individuales en la sensibilidad a la recompensa y la conectividad funcional de las áreas neurales asociadas al BAS, observadas durante el estado de reposo en un grupo de hombres jóvenes. Para ello, utilizaremos áreas relacionadas con el BAS de la investigación de morfometría basada en el vóxel de Barrós-Loscertales y cols. (2006) en las que encontraron correlaciones negativas entre el volumen de sustancia gris del estriado derecho, el estriado izquierdo y el cortex frontal superior derecho con las puntuaciones en SR. Nuestra hipótesis es que las diferencias individuales en sensibilidad a la recompensa se asociarán a diferencias en la conectividad funcional entre las diversas áreas que han mostrado reducciones en sustancia gris.

\section{Método}

\section{Muestra}

Han participado en este experimento 88 sujetos, varones, estudiantes, con edades comprendidas entre 18 y 30 años (media $=21,67$; desviación típica $=2,77$ ). Se obtuvo el consentimiento informado de cada sujeto antes de la participación y recibieron, por ella, una compensa- 
ción económica. El proyecto de investigación fue aprobado por el Comité Ético de la Universidad Jaume I.

\section{Instrumentos}

Los participantes rellenaron el Cuestionario de Sensibilidad al Castigo y Sensibilidad a la Recompensa (SCSR) (Torrubia y cols., 2001). Es un cuestionario de autoinforme que consta de 48 ítems con un formato de respuesta dicotómica (Sí/No). Se compone de dos subescalas de 24 ítems cada una. La escala de sensibilidad al castigo (ítems: 1, 3, 5, 7, 9, 11, 13, 15, 17, 19, 21, 23, $25,27,29,31,33,35,37,39,41,43,45,47)$ se relaciona con el sistema de ilnhibición conductual, BIS (evitación conductual y evitación pasiva en situaciones relacionadas con la posibilidad de obtener consecuencias negativas o novedad, preocupación vinculada al daño por castigos o fracasos). La escala de sensibilidad a la recompensa (ítems: 2, 4, 6, 8, 10, 12, 14, 16, 18, 20, $22,24,26,28,30,32,34,36,38,40,42,44,46,48)$ se relaciona con el sistema de activación conductual, BAS (conductas dirigidas a la obtención de refuerzos tales como dinero, poder, sexo y búsqueda de sensaciones).

\section{Análisis de las imágenes}

Las imágenes fueron adquiridas en un escáner 1,5 T (Siemens Avanto). Las imágenes de RMf se obtuvieron en estado de reposo en el que los participantes simplemente fueron instruidos en mantenerse relajados, intentando no pensar en nada y sin llegar a dormirse. La muestra de este trabajo se obtuvo de una base de datos de distintos estudios por lo que algunos sujetos realizaron la tarea con ojos cerrados y otros con ojos abiertos mirando a un punto fijo. Los participantes se colocaron en una posición supina en el escáner de RM, y sus cabezas fueron inmovilizadas con los amortiguadores para reducir los artefactos en movimiento. Para el RMf-ER, un total de 270 volúmenes fueron registrados por encima de los 9 minutos usando un gradiente de eco T2*-secuencia de imágenes ecoplanar ponderada (TR $=2000 \mathrm{~ms}$; $\mathrm{TE}=48$ $\mathrm{ms}$; matriz, $64 \times 64$, tamaño de voxel = 3,5 x 3,5 mm, ángulo flip $=900$; grosor de corte $=4$ $\mathrm{mm}$; gap $=0,8 \mathrm{~mm}$. Adquirimos 24 cortes axiales intercalados paralelos al plano de la comisura anterior-posterior que cubre la totalidad cerebral. Antes de las secuencias de RMf, un T1 estructural de alta resolución de secuencia MPRAGE ponderada fue adquirido (TR $=2200 \mathrm{~ms}$; TE $=3,8$ ms; matriz $=256 \times 256 \times 160$; voxel size $=1 \times 1 \times 1 \mathrm{~mm}$ ). Los conjuntos de datos RMf-ER fueron procesados mediante el programa Data Processing Assistant for Resting-State fMRI (DPARSFA, http://rfmri.org/DPARSF) (Chao-Gan y Yu-Feng, 2010) donde el preprocesado está basado en algunas funciones del programa Statistical Parametric Mapping (v.8 SPM Wellcome Trust Centro de Neuroimagen, Londres, Reino Unido) y los análisis de conectividad en el programa REST rs-fMRI (http://www.restfmri.net).

Las seed seleccionadas para nuestro estudio fueron extraídas de un estudio de morfometría basada en el vóxel. En este estudio se encontraron correlaciones negativas entre el volumen de sustancia gris del estriado derecho, el estriado izquierdo y el cortex frontal superior derecho con las puntuaciones en SR (Barrós-Loscertales y cols., 2006). Las seeds en el espacio MNI fueron el cuerpo estriado derecho $(4,12,10)$, el caudado derecho $(17,14,14)$, el estriado izquierdo $(-24,9,14)$, el caudado izquierdo $(-18,15,10)$, globo pálido izquierdo $(-17,-4,9)$ y el cortex superior frontal derecho $(13,48,29)$.

El análisis de la conectividad funcional basado en voxel semilla se realizó para las seeds anteriormente definidas. Se llevó a cabo una correlación lineal pareada (correlación de Pearson) 
entre las series temporales de los voxels de cada seed, obteniendo para cada par de seeds un valor de correlación. Los valores $r$ obtenidos para cada sujeto se normalizaron a valores $z$ usando la transformación z de Fisher, para su posterior análisis estadístico.

\section{Análisis estadísticos}

Los valores $z$ obtenidos del análisis de conectividad funcional fueron correlacionados con los valores de SR, usando el programa estadístico IBM SPSS (versión 21) con el fin de estudiar la relación entre las puntuaciones del test y la conectividad funcional de las zonas de interés durante el estado de reposo.

\section{Resultados}

Los resultados de nuestra investigación indican que la media de las puntuaciones en sensibilidad a la recompensa de nuestra muestra es $=11,97$ y la desviación típica $=4,98$. Encontramos una correlación significativa positiva entre la conectividad funcional del estriado dorsal izquierdo con el derecho y las puntuaciones en sensibilidad a la recompensa, con una correlación de Pearson = 0,432 significativa al nivel 0,000; como se puede apreciar en la Figura 1. No se han encontrado correlaciones significativas entre la conectividad funcional del resto de clústers, estriado dorsal izquierdo con la corteza frontal superior derecha y estriado dorsal derecho con esta última, y puntuaciones en sensibilidad a la recompensa. Todo ello queda reflejado en la Tabla 1.

Tabla 1

Estadísticos y correlaciones de las variables del estudio

\begin{tabular}{lcccccc}
\hline & $\begin{array}{c}\text { Correlacíón } \\
\text { en SR }\end{array}$ & Media & Desv. típ. & Mín. & Máx. & N \\
\hline $\begin{array}{l}\text { Puntuación SR } \\
\text { Conectividad funcional: }\end{array}$ & - & 11,97 & 4,98 & 3 & 23 & 88 \\
$\begin{array}{l}\text { Estriado derecho con estriado } \\
\text { izquierdo }\end{array}$ & $0,432^{* *}$ & 0,177 & 0,154 & $-0,161$ & 0,47 & 88 \\
$\begin{array}{l}\text { Estriado izquierdo con corteza } \\
\text { prefrontal superior derecha }\end{array}$ & 0,104 & 0,007 & 0,131 & $-0,292$ & 0,387 & 88 \\
$\begin{array}{l}\text { Estriado derecho con corteza } \\
\text { prefrontal superior derecha }\end{array}$ & 0,145 & 0,01 & 0,138 & $-0,322$ & 0,39 & 88 \\
\hline
\end{tabular}




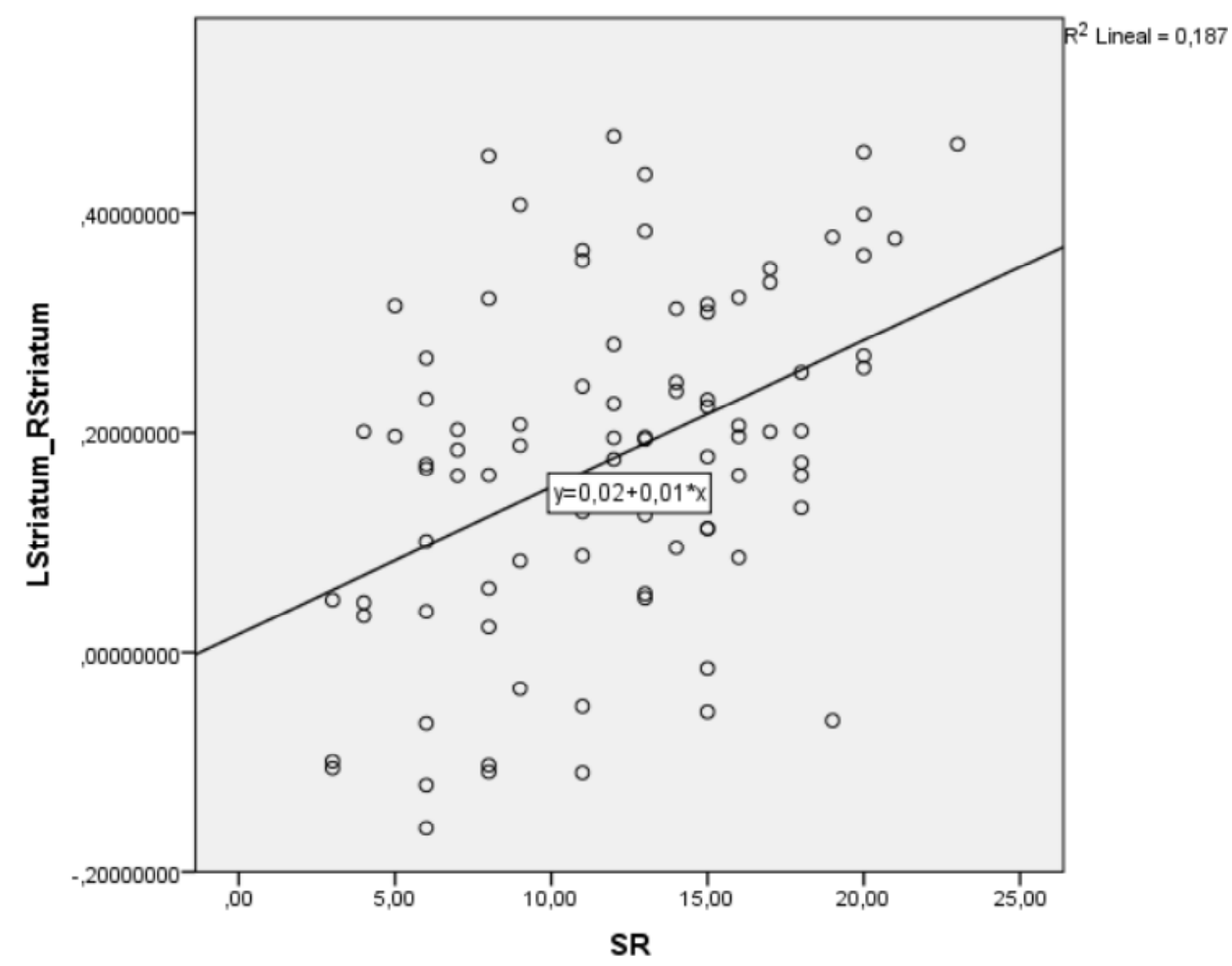

Figura 1. Asociación entre las puntuaciones en sensibilidad a la recompensa (SR) y los valores de la correlación entre estriado derecho e izquierdo en estado de reposo (LStriatum_RStriatum).

\section{Discusión y conclusiones}

Los resultados del presente estudio han demostrado una correlación positiva en la activación producida entre las áreas izquierda y derecha del estriado dorsal en estado de reposo y las puntuaciones en el cuestionario de sensibilidad a la recompensa en hombres jóvenes. Observamos qué a mayor activación en la conectividad funcional de estas áreas, mayores puntuaciones en SR.

Estos datos continúan en la senda de otros estudios que implican a los ganglios basales en las diferencias individuales en el procesamiento de la recompensa. Se ha visto como sujetos con puntuaciones altas en escalas asociadas al BAS muestran una mayor activación del estriado ventral y el mesencéfalo durante la anticipación de recompensas monetarias (Carter, Macinnes, Huettel y Adcock, 2009; Costumero, 2013), y durante la recepción de éstas (Camara, RodriguezFornells y Münte, 2010; Cservenka, Herting, Seghete, Hudson y Nagel, 2012). Además, una alta reactividad del BAS se ha relacionado también con un incremento de la activación del estriado ventral y mesencéfalo ante la presentación de imágenes de comidas apetitivas (Beaver y cols., 2006). Como en la presente investigación, la SR se ha asociado positivamente con la actividad del estriado ventral (Avila y cols., 2012).

El estriado dorsal se relaciona con el sistema de recompensa, por lo tanto, estos resultados apoyan la participación de circuitos fronto-estriatales en el sistema de activación conductual (De- 
pue y Collins, 1999; Pickering y Gray, 2001). Esta área es importante en la selección e iniciación de la conducta motivada y en el aprendizaje de asociaciones respuesta-consecuencia (Balleine, Delgado y Hikosaka, 2007); en otras palabras, en el procesamiento de la información relacionada con la recompensa. La actividad del estriado juega un importante papel en el procesamiento de recompensas primarias y secundarias, por consiguiente, parece que pueda influir y guiar el comportamiento mediante la codificación de las propiedades afectivas de un estímulo (BarrósLoscertales y cols., 2006); interviniendo dentro de los circuitos fronto-estriatales en la regulación de la conducta al establecer patrones aprendidos por repetición.

En nuestro estudio, encontramos diferencias individuales en la conectividad del estriado que se relaciona con el factor de personalidad de Gray de sensibilidad a la recompensa, empleando la RMF-ER. En otro estudio de personalidad en el que también se empleaba la RMF-ER, se encontraron resultados similares, una mayor aleatoriedad de las series temporales del estriado ventral en sujetos con alta SR (Hahn y cols., 2012). Por lo tanto, tal y como comentó Harmelech, los patrones de correlación entre las fluctuaciones de actividad espontánea durante el estado de reposo podrían reflejar rasgos de personalidad (Harmelech y Malach, 2013). Ya que los patrones de activación espontáneos, producidos durante el reposo, correspondería a patrones de activación que se producen durante la ejecución de tareas típicas en el día a día; observándose las diferencias individuales en ese estado de reposo.

En conclusión, hemos empleado la novedosa condición experimental de estado de reposo en la RMf, mostrando la correlación entre la conectividad funcional del estriado dorsal derecho e izquierdo y la activación del BAS. Demostrando que las diferencias individuales en la conectividad funcional del estriado tienen su repercusión en las puntuaciones de sensibilidad a la recompensa.

\section{Referencias bibliográficas}

Aertsen, A. M., Gerstein, G. L., Habib, M. K. y Palm, G. (1989). Dynamics of neuronal firing correlation: modulation of «effective connectivity». Journal of Neurophysiology., 61, 900-917.

Avila, C., Garbin, G., Sanjuán, A., Forn, C., Barrós-Loscertales, A., Bustamante, J. C. et al (2012). Frontostriatal response to set switching is moderated by reward sensitivity. Social Cognitive and Affect Neuroscience, 7, 423-430.

Balleine, B. W., Delgado, M. R. y Hikosaka, O. (2007). The role of the dorsal striatum in reward and decision-making. Journal of Neuroscience, 27, 8161-8165.

Barrós-Loscertales, A., Meseguer, V., Sanjuán, A., Belloch, V., Parcet, M., Torrubia, R. et al. (2006). Striatum gray matter reduction in males with an overactive behavioral activation system. European Journal of Neuroscience, 24, 2071-2074.

Beaver, J. D., Lawrence, A. D., Van Ditzhuijzen, J., Davis, M. H., Woods, A. y Calder, A. J. (2006). Individual differences in reward drive predict neural responses to images of food. Journal of Neuroscience, 26, 5160-5166.

Berridge, K. C. y Kringelbach, M. L. (2008). Affective neuroscience of pleasure: reward in humans and animals. Psychopharmacology (Berl), 199, 457-480.

Biswal, B., Yetkin, F. Z., Haughton, V. M. y Hyde, J. S. (1995). Functional connectivity in the motor cortex of resting human brain using echo-planar MRI. Magnetic Resonance in Medicine, $34,537-541$.

Camara, E., Rodriguez-Fornells, A. y Münte, T. F. (2010). Microstructural brain differences predict functional hemodynamic responses in a reward processing task. Journal of Neuroscience, 30, 11398-11402. 
Carter, R. M., Macinnes, J. J., Huettel, S. A. y Adcock, R. A. (2009). Activation in the VTA and nucleus accumbens increases in anticipation of both gains and losses. Frontiers in Behavioral Neuroscience, 3, 21.

Chao-Gan. Y. y Yu-Feng, Z. (2010). DPARSF: a MATLAB toolbox for «pipe-line» data analysis of resting-state fMRI. Frontiers in System Neuroscience, 4,13.

Corr, P. J. (2008a). The Reinforcement Sensitivity Theory of Personality. Cambridge: Cambridge University Press.

Corr, P. J. (2008b). Reinforcement Sensitivity Theory (RST): introduction. P.J. Corr, ed. In The Reinforcement Sensitivity Theory of Personality (pp. 1-43). Cambridge: University Press.

Costumero, V. (2013). Función, conectividad cerebral y diferencias individuales en el procesamiento de recompensas. (Tesis doctoral). Universidad Jaume I. Castellón.

Cservenka, A., Herting, M. M., Seghete, K. L. M., Hudson, K. A. y Nagel, B. J. (2012). High and low sensation seeking adolescents show distinct patterns of brain activity during reward processing. Neuroimage, 66, 184-193.

Depue, R. A. y Collins, P. F. (1999). Neurobiology of the structure of personality: dopamine, facilitation of incentive motivation, and extraversion. Behavioral and Brain Sciences, 22, 491517; discussion 518- 69.

Friston, K. J., Frith, C. D., Liddle, P. F. y Frackowiak, R. S. (1993). Functional connectivity: the principal-component analysis of large (PET) data sets. Journal of Cerebral Blood Flow \& Metabolism, 13 (1), 5-14.

Gray, J. A. (1970). The psychophysiological basis of introversion-extraversion. Behavior Research and Therapy, 8, 249-266.

Haber, S. N. y Knutson, B. (2010). The reward circuit: linking primate anatomy and human imaging. Neuropsychopharmacology, 35, 4-26.

Hahn, T., Dresler, T., Ehlis, A. C., Pyka, M., Dieler, A. C., Saathoff, C. et al (2012). Randomness of resting-state brain oscillations encodes Gray's personality trait. Neuroimage, 59, 1842 1845.

Harmelech, T. y Malach, R. (2013). Trends in Cognitive Sciences, 17 (12), 606-615.

McClure, S. M., York, M. K. y Montague, P. R. (2004). The neural substrates of reward processing in humans: the modern role of FMRI. Neuroscientist, 10, 260-268.

Pickering, A. D. y Gray, J. A. (2001). Advances in Individual Differences Research. In Eliasz, A. y Angleitner, A. (Eds). Dopamine, appetitive reinforcement, and the neuropsychology of human learning: an individual differences approach (113-149) Germany: Lengerich.

Pickering, A. D. y Smillie, L. D. (2008). The behavioural activation system: Challenges and opportunitie.P.J. Corr, ed. In The Reinforcement Sensitivity Theory of Personality (120-153). Cambridge: University Press.

Salvador, R., Suckling, J., Coleman, M. R., Pickard, J. D., Menon, D. y Bullmore, E. (2005). Neurophysiological architecture of functional magnetic resonance images of human brain. Cerebral Cortex, 15 (9), 1332-1342.

Torrubia, R., Ávila, C., Moltó, J. y Caseras, X. (2001). The sensitivity to punishment and sensitivity reward questionnaire (SPSRQ) as a measure of Gray's anxiety and impulsivity dimensions. Personality and Individual Differences, 31, 837-862. 